\title{
Blind Estimation of Wide-band Frequency-Hopping Signal Based On Compressed Numeral Characteristics
}

\author{
Xun $\mathrm{Lu}^{1, \mathrm{a}}$, Lichun $\mathrm{Li}^{2, \mathrm{~b}}$ and Zhong $\mathrm{Liu}^{3, \mathrm{C}}$ \\ 1,2,3 I.T.Institute of Zhengzhou, Henan Province, China \\ a stilluxun@163.com, b leetracy@139.com, 'liuzhongsky@qq.com
} Keywords: compressed sensing; Frequency-Hopping signal; parameter estimation; compressed
numeral characteristics.

\begin{abstract}
In order to make a precise and quick estimation of the wide-band Frequency-Hopping(FH) signal, a blind parameter estimation using numeral characteristics of compressive samplings is proposed. First study the compressed numeral characteristics (CNC) of the signal on the premise of non-reconstructing the original signal to detect the changes of signal frequency. Then reconstruct these data sections to get its frequency domain coefficients. Estimations could be achieved with hopping frequency, frequency-hopping periods and the frequency-hopping moments based on the frequency domain coefficients. Simulation results show that this algorithm can achieve the same error conditions as Wigner algorithm with Gaussian white noise when SNR is 10dB and compress rate is $1 / 4$. And the number of sampling points of processing required for a quarter of the Wigner algorithm. So this algorithm has the advantages of low computing complexity and better real-time performance compared with then traditional algorithm using time-frequency characteristics.
\end{abstract}

\section{Introduction}

With a lot of advantages such as high security, good anti-jam performance and low probability of interception, Frequency-Hopping (FH) signals have been widely used in the field of military and civilian communications. The parameter estimation of the unknown FH signal mixed with noise means to estimate the FH period, FH time and the carrier frequency for the subsequent demodulation decryption or track interference.

Traditional time-frequency analysis method is usually divided into the short-time Fourier Transform $^{[1]}$, Wigner-Ville distribution ${ }^{[2]}$, Wavelet Transform ${ }^{[3]}$ and so on. In recent years, the FH signals have the development trends of the high frequency band, large bandwidth. According to the Nyquist sampling theorem, it needs two times the bandwidth of the sampling rate to ensure undistorted sampling $\mathrm{FH}$ signal, thus the difficulty of the FH signal sampling and processing of computational complexity has greatly increased, so that the efficiency of the traditional signal processing reduces.

As the Compressed Sensing ${ }^{[4]}$ technology puts out that CS can sample the sparse or compressive signals by the sampling rate far below than the Nyquist sampling rate and ensure that there is no loss of information in the signals. The general signal processing way is to accurately reconstruct of sampling values, and then estimate the parameter using the traditional methods. But the accurate reconstruction needs large computation which is not conducive to real-time signal processing. In order to achieve the detection, estimation and classification purposes, related literatures in recent years have studied how to extract the information directly from the compressed sampling values. Sampling the FH signals at a relatively low rate by using CS technology, and then estimate the signal parameter on the premise of dis-reconstruct the original signal. Reference [5] has studied a FH signal synchronization algorithm based on subspace. This algorithm needs to know FH period in advance and not consider the sparsity of FH signal in the frequency domain, then it limit the practical application. Reference [6] has studied a slide compressive parameter estimation algorithm. This algorithm should know FH period in advance, and reconstruct the frequency domain coefficients through the OMP algorithm, time-effectiveness is yet to be improved as the computational cost is large. 
In this paper, on the premise of not completely reconstruct the original signal, by the use of $\mathrm{FH}$ signals' sparsity in the frequency domain, a parameter blind estimation(CS-PBE) algorithm based on CNC has been proposed. This algorithm can accurately estimate the hopping time and carrier frequency. This algorithm uses compressed sampling value directly, and can satisfy the real-time processing of FH signals because of the small computational complexity and low algorithm complexity. Paper structure is as follows: the first part is the introduction; the second part introduces the CS technology, FH signal model and parameter estimation model; the third part introduces CS-PBE algorithm; the simulation validation is processed in the fourth part; the fifth part summarizes the whole thesis and makes conclusions.

\section{Mathematical Model}

\section{A. Compressed Sensing Technology}

Compressed sensing theory refers to using a compressed projection matrix of the sparse signal (sparse in a transform domain) mapping, without losing the information in the original signal when project the high-dimensional signals into low dimensional space, then reconstruct the original signal through an optimization problem. Mainly includes three parts: sparse representation, linear mapping and signal reconstruction.

a) Sparse Representation

The signals have the sparse representation is the premise of the application of CS. Define an N dimension discrete signal $\mathrm{x}$, it can be expressed as a linear combination of a set of standard orthogonal basis form:

$$
x=\sum_{i=1}^{N} s_{i} \psi_{i} \quad \text { or } \quad x=\Psi s
$$

Let $\Psi=\left[\psi_{1}, \psi_{2}, \cdots, \psi_{N}\right]$ be a set of standard orthogonal basis or frame. It can be set as Fourier orthogonal basis and wavelet basis. Let $\psi_{i}$ be $N \times 1$ column and $s$ be the coefficient vector of signal $\boldsymbol{x}$. If the vector $\boldsymbol{s}$ have $K$ nonzero elements, all other elements are zero, then we can consider $\boldsymbol{s}$ as the sparse representation for signal $\boldsymbol{x}$ in orthogonal basis $\boldsymbol{\Psi}$, and the sparse degree is $K$.

b) Linear Mapping

The principle of compressed sensing is to linear map high-dimensional signals to low dimensional space, it can greatly reduce the amount of data signal processing. By constructing a $M \times N$ dimension compressive measurement matrix $\Phi$, we can get the linear measurement value of the signal $x$ :

$$
y=\Phi x
$$

Let $y \in R^{M \times 1}$ be the compressed sampling values got from $x$. Equation is the basic process of compressive sensing. Put equation into equation:

$$
y=\Phi \Psi s=\Theta s
$$

Let $\Theta=\Phi \Psi \in R^{M \times N} \quad(M<N)$, and we call it the sensing matrix. CS can achieve the purpose of reducing the amount of processing data by sensing compressed sampling values $y$. In order to accurately recover the original signal from compressed sampling values, the sensing matrix $\Theta$ need to satisfy the Restricted Isometry Property (RIP) conditions ${ }^{[7]}$. The measurement matrix commonly used includes Gaussian random matrix and Bernoulli random matrix which can satisfy RIP conditions with great probability.

c) Signal Reconstruction

The reconstruction of sparse signals $\boldsymbol{x}$ can be regarded as solving the following optimization problem:

$$
\min _{\tilde{x} \in R^{N}}\|\tilde{x}\|_{l_{0}} \quad \text { s.t. } \Phi \Psi \tilde{x}=y
$$

There are two main ways to solve this problem: Basis Pursuit (BP) ${ }^{[8]}$ and Orthogonal Matching Pursuit (OMP) ${ }^{[9]}$. The BP algorithm uses $l_{1}$-norm optimization to recover signal $\boldsymbol{x}$. OMP algorithm 
means to look for the biggest correlation with residual and has selected atoms library orthogonalization, and then find out residual in each iteration process. After several iterations, we can approximate original sparse signal expressed as a linear combination of these atoms.

B. Analysis of Wide-band FH Signal's Sparse

FH signal model are as follows:

$$
x(t)=\sum_{n=1}^{N} s_{n}(t)+n(t)=\sum_{n=1}^{N} a_{n}(t) e^{j(\omega(t) t+\varphi(t))}+n(t)
$$

Where frequency and phase are the time-varying functions, $\ldots-\dot{U}$ is white Gaussian noise.

For wide-band FH signal, it is sparse in a single FH window. Use Gaussian random measurement matrix to block sample the FH signal. Then we can get signal hopping range of conversion time through comparing with CNC. Two types of FH signal observation model are as follows:

1. When observation period locates in a FH period, sparse degree of the signal in the observation period is 1 in ideal condition. As shown in figure 1:

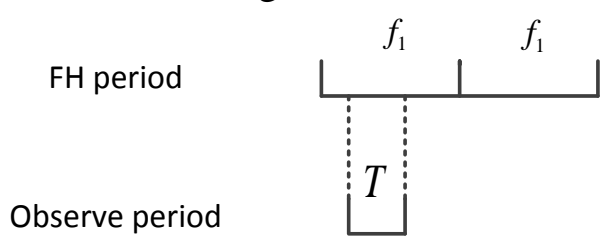

Figure 1 1-sparse signal observation model

2. When observation period locates between the two FH periods, sparse degree of the signal in the observation period is 2 in ideal condition. As shown in figure 2:

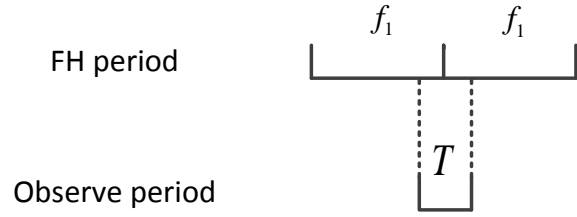

Figure 2 2-sparse signal observation model

\section{Discrete Fourier Transform(DFT) of FH Signal}

Single hopping signal can be seen as discrete sine signals, it can be expressed as:

$$
x(n)=A \cos \left(\frac{2 \pi f}{f_{s}} n+\varphi\right)
$$

Where $A$ is amplitude, $f$ is carrier frequency, $f_{s}$ is sampling frequency, $\varphi$ is primary phase. Conduct DFT on the $\mathrm{N}$ points long signal $x(n)$ to get $X(k)$ :

$$
X(k)=\sum_{n=0}^{N-1} x(n) e^{-j \frac{2 \pi}{N} k n}=\sum_{n=0}^{N-1} A \cos \left(\frac{2 \pi f}{f_{s}} n+\varphi\right)\left[\cos \left(\frac{2 \pi}{N} k n\right)-j \sin \left(\frac{2 \pi}{N} k n\right)\right]
$$

Through product of summing equation, we can obtain:

$$
\begin{aligned}
X(k) & =\sum_{n=0}^{N-1} \frac{A}{2} \cos \left(2 \pi\left(\frac{f}{f_{s}}+\frac{k}{N}\right) n+\varphi\right)+\sum_{n=0}^{N-1} \frac{A}{2} \cos \left(2 \pi\left(\frac{f}{f_{s}}-\frac{k}{N}\right) n+\varphi\right) \\
& -j \sum_{n=0}^{N-1} \frac{A}{2} \sin \left(2 \pi\left(\frac{f}{f_{s}}+\frac{k}{N}\right) n+\varphi\right)+j \sum_{n=0}^{N-1} \frac{A}{2} \sin \left(2 \pi\left(\frac{f}{f_{s}}-\frac{k}{N}\right) n+\varphi\right)
\end{aligned}
$$

Substitute $N=m f_{s}$ into equation: 


$$
\begin{aligned}
X(k) & =\sum_{n=0}^{N-1} \frac{A}{2} \cos \left(\frac{2 \pi}{N}(m f+k) n+\varphi\right)+\sum_{n=0}^{N-1} \frac{A}{2} \cos \left(\frac{2 \pi}{N}(m f-k) n+\varphi\right) \\
& -j \sum_{n=0}^{N-1} \frac{A}{2} \sin \left(\frac{2 \pi}{N}(m f+k) n+\varphi\right)+j \sum_{n=0}^{N-1} \frac{A}{2} \sin \left(\frac{2 \pi}{N}(m f-k) n+\varphi\right) \\
& =X_{\mathrm{Re} 1}(k)+X_{\mathrm{Re} 2}(k)-j X_{\mathrm{Im} 1}(k)+j X_{\mathrm{Im} 2}(k)
\end{aligned}
$$

If $m f+k \neq 0$, we know that the sinusoidal signals in the sum of $X_{\mathrm{Re} 1}(k)$ and $X_{\mathrm{Im} 1}(k)$ must be based on N period. So the sum from 0 to $N-1$ is zero. Similarly, if $m f-k \neq 0$, the sum of $X_{\operatorname{Re} 2}(k)$ and $X_{\operatorname{Im} 2}(k)$ must be zero too. To make $m f+k$ to be zero, there will be $f=k=0$; To make $m f-k$ to be zero, there will be $m f=k$.

Through the analysis above, when $m f-k$ is zero, the $X(k)$ will have value as:

$$
|X(k)|=\frac{A N}{2}
$$

Where $k=f /\left(f_{s} / N\right)$.

By the use of numerical value and its position in the original signal, we can accurately estimate the original hopping time of FH signal and carrier frequency.

\section{Blind Estimation of FH Signal Based On CNC}

Consider about the FH signal model in equation, get the compressed sampling of signal according to the equation. Choose block sampling method in this algorithm considering that $\mathrm{FH}$ signal corresponding to different sparse model when the frequency window locates in different space.

For an $\mathrm{N}$ dimension discrete signal $\boldsymbol{x}$, we construct a $M_{1} \times N_{1}$ dimension measurement matrix. Let $N_{1}=2048$, The $i$ th compressed sampling value is:

$$
y_{i}=\Phi x\left[1+(i-1) N_{1}, \cdots, i N_{1}\right]
$$

Where $y=\left[y_{1}^{T}, y_{2}{ }^{T}, \cdots, y_{\left[N / N_{1}\right]}{ }^{T}\right]^{T}$, and $\left[N / N_{1}\right]$ is the truncating operation.

According to two sparse signal models of FH signal above, provide blind estimation algorithm of FH signal conversion time and carrier frequency.

A. Rough Estimation of Conversion Time Based On CNC

If observation period locate in a single hopping period as shown in figure 1, calculate inner product of compressed measurements and each column in holographic matrix considering about the 1-sparse feature of the signal:

$$
\mu=\Theta^{H} y
$$

Where $\mu_{i}=<\Theta_{i}^{H}, y>, \Theta_{i}^{H}$ is the $i$ th column vector of holographic matrix and $i=1,2, \cdots, M_{1}$. The physical meaning it represented is the relevance of compressed measurements and each column of the holographic matrix. Through the maximization of $\mu_{i}$, choose the largest column that makes $\mu_{i}$ maximal. Take the position of the column vector as Compressed Numeral Characteristics:

$$
G_{i}=\arg \max _{i}\left[<\Theta_{i}^{H}, y_{i}>\right]
$$

Then we can judge signal hopping time range through the difference of CNC. It corresponds to 2-sparse signal observation model shown in figure 2.

B. Accurate Estimation of Hopping Time

Use OMP algorithm to reconstruct the two compressed measurements which are got from rough estimation above, then we can obtain two coefficients in frequency domain. Through the derivation of FH signal's DFT, we know that position of the coefficients in frequency domain correspond to the carrier frequency, and coefficient values correspond to the length of the signal located in the original signal for a certain frequency. Then we can estimate the carrier frequency and hopping time to achieve the purpose of accurate estimation. 
This algorithm only needs to handle little compressed sampling values to complete the parameter estimation of $\mathrm{FH}$ signal under the premise of without knowing any $\mathrm{FH}$ parameters. Reduce the amount of data processing greatly, and improve the real-time performance of the algorithm. Algorithm steps are as follows:

1. Block compressed sample of the original signal:

$$
y_{i}=\Phi \Psi_{f} S_{i}=\Theta s\left[1+(i-1) N_{1}, \cdots, i N_{1}\right]
$$

Where $\Psi_{f} \in R^{N_{1} \times N_{1}}$ is Discrete Fourier orthogonal sparse matrix, and $s_{i}=s\left[1+(i-1) N_{1}, \cdots, i N_{1}\right]$ is $i$ th coefficient vector.

2. Calculate the inner product of $y_{i}$ and each column in $\Theta$ :

$$
\mu_{i}=<\Theta_{i}^{H}, y_{i}>
$$

3. Calculate the Compressed Numeral Characteristics of the signal:

$$
G_{i}=\arg \max _{i}\left[<\Theta_{i}^{H}, y_{i}>\right]
$$

4. Determine two compressed sampling values as the hopping period by comparing the difference between $G_{i}$. Then reconstruct signals from these two sampling values to obtain coefficients of these two signals in frequency domain: $X_{f_{1}}\left(k_{1}\right)$ and $X_{f_{2}}\left(k_{2}\right)$. Where $k_{1}=f_{1} /\left(f_{s} / N_{f_{1}}\right)$ and $k_{2}=f_{2} /\left(f_{s} / N_{f_{2}}\right) . N_{f_{1}}$ and $N_{f_{2}}$ represent the signal length corresponding to the frequency.

5. Consider about the coefficients of signals in frequency domain: $\left|X_{f_{1}}(k)\right|=\frac{A N_{f_{1}}}{2}$ and $\left|X_{f_{2}}(k)\right|=\frac{A N_{f_{2}}}{2}$. Where $\left|X_{f_{1}}(k)\right|$ and $\left|X_{f_{2}}(k)\right|$ correspond to the amplitude of FFT for different frequency.

The algorithm flow chart of Blind Estimation Algorithm of Wide-band FH signal based on Compressed Numeral Characteristics shows in figure 3:

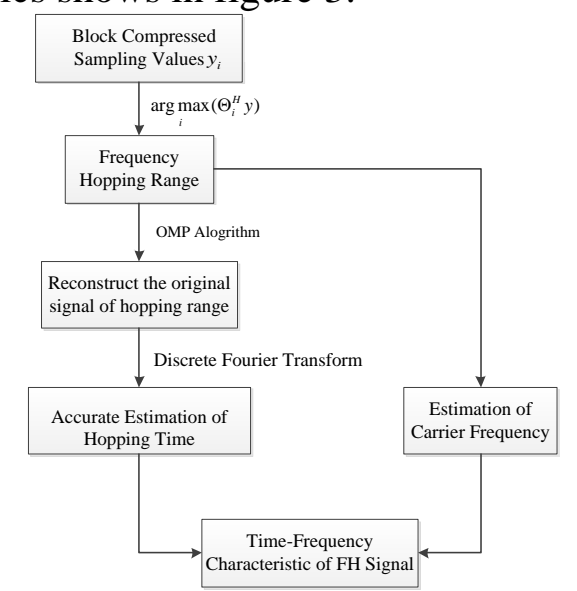

Figure 3 Flow chart of CS-BPE algorithm

\section{Simulation and Analysis of Algorithm}

The compressed samples $y$ are obtained by the block compressed sample for the original signal with measurement matrix $\Phi$ ( $\Phi \in R^{M_{1} \times N_{1}}, N_{1}=2048$ ). Where $N_{1}$ is the single sampling point, the elements of $\Phi$ are all consistent with the Gaussian distribution $\mathrm{N}(0,1 / M)$. Sparse matrix $\Psi$ is the standard discrete Fourier orthogonal basis.

Original signal parameter settings are as follows: hopping period is $T=1 \mathrm{~ms}$, Nyquist sampling rate is $F_{s}=20 \mathrm{MHz}$. Each FH cycle includes 10000 sampling points. Modulation model is BFSK, the symbol rate is $2000 \mathrm{~B}$, each hopping cycle sends a code element. The total bandwidth of FH signal is 8 
$\mathrm{MHz}$, frequency interval is $1 \mathrm{MHz}$. The duration of the first hopping is randomly generated in $0 \sim T$. The FH signal's STFT graph is shown in Figure 4:

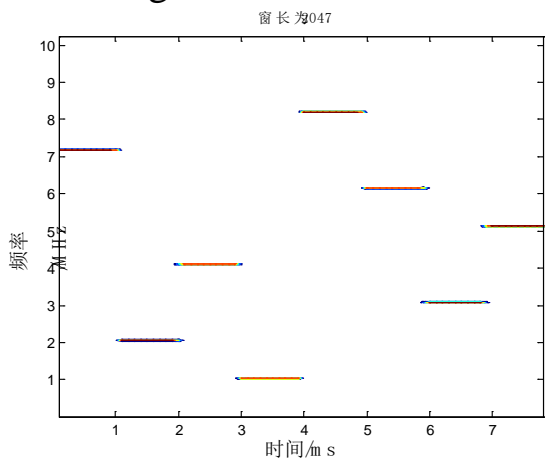

Figure 4 FH signal time-frequency figure

Definite normalized mean square error $\operatorname{NMSE}(\hat{f})$ of carrier frequency estimation $\hat{f}$ is:

$$
\operatorname{NMSE}(\hat{f})=\frac{1}{N_{T}} \sum_{i=1}^{N_{T}}\left(\frac{\hat{f}_{i}-f_{i}}{f_{i}}\right)^{2}
$$

Algorithm uses expectation of the ratio between estimation error and single FH cycle square to represent FH hopping time's estimation error:

$$
E\left(\Delta_{K}\right)=E\left(\frac{|\hat{K}-K|}{N}\right)^{2}
$$

A. Carrier Frequency Estimation Simulation

The NMSE of using this algorithm to estimate carrier frequency under different signal-to-noise ratio and compression ratio is shown in figure 5 :

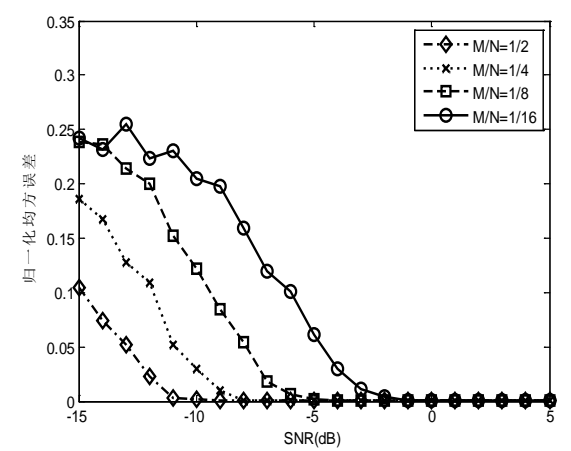

Figure 5 NMSE of carrier frequency estimation under different SNR and compression ratio

As shown above, as the signal-to-noise ratio SNR and the compression ratio $\mathrm{M} / \mathrm{N}$ change, performance of carrier frequency estimation will be better. Under the condition of $M / N=1 / 2$, and SNR is above $-10 \mathrm{~dB}$, algorithm achieve the optimal carrier frequency estimate.

\section{B. FH Signal Hopping Time Estimation Simulation}

Using this algorithm of estimating hopping time to make comparison with the Wigner method under different SNR conditions.

We can regard the biggest transformation frequency of single hopping period as the hopping time estimation. For signal above, respectively conduct two algorithms by 1000 monte-carlo experiments independently. The compression ratio of block compression in the process of sampling in this paper is $M / N=1 / 4$.Sampling points in single sampling time is $N=2048$. Different estimated error of hopping time under different SNR conditions is shown in figure 6. It can be seen from the simulation results that CS-BPE algorithm is better than Wigner method in the case of low signal noise ratio because that the Wigner algorithm does not take into account the removal of cross terms. With the improvement of signal-to-noise ratio, two algorithms of parameter estimation error are reduced 
accordingly. By the condition of $S N R>4 d B$, the error of the Wigner method is close to the algorithm in this paper.

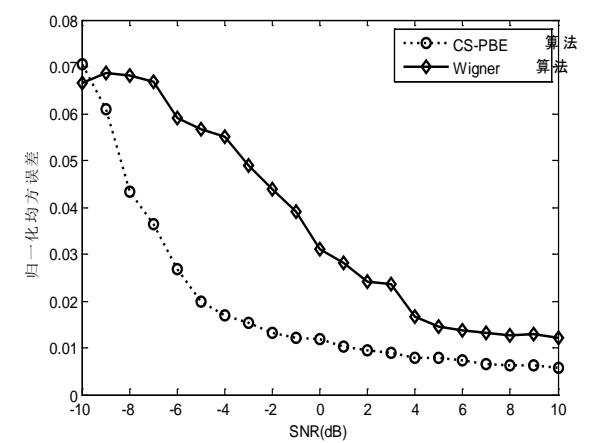

Figure 6 Hopping time error comparison under different SNR

As is shown in figure 6, the simulation error of this algorithm is lower than Wigner algorithm.

\section{Conclusion}

This paper puts forward a FH signal blind estimation algorithm based on compressed numeral characteristics. First of all, find out the location of the frequency changes through the study of compressed numeral characteristics under the premise of not to reconstruct the original signal. And then reconstruct the data segment to get the coefficients in frequency domain. At last, estimate carrier frequency and FH time according to the location and size of the coefficients in frequency domain. The simulation results verify the performance of the algorithm, because only a small piece of data is needed for estimation, the algorithm performance is better than the traditional algorithm, and has a huge advantage in computing complexity. Algorithm in this paper does not need to know a prior information of FH signal, without having to completely reconstruct the original signal, so that greatly simplify the procedure of signal processing, improve the time-validity of the FH signal parameter estimation.

\section{References}

[1] Gabor D. Theory of communication[J]. IEE, 1946, 93: 429-457.

[2] Cohen L. Time-Frequency Analysis[M]. New Jersey: Prentice Hall, 1995.

[3] B Boashash. Time-Frequency Signal Analysis and Processing: A Comprehensive Reference [M]. Oxford, UK:Elsevier Science, 2003.

[4] Candès E. Compressive sampling [C]//Proceedings of the International Congress of Mathematicians, Madrid, Spain, August 22-30, 2006, 3, 1433-1452.

[5] Yuan J, Tian P, Yu H. Subspace compressive frequency estimation of FH signal[C]//Wireless Communications, Networking and Mobile Computing, 2009. WiCom'09. 5th International Conference on. IEEE, 2009: 1-4.

[6] Zhang Chunlei, Li Lichun, Wang Daming. An estimation algorithm for broadband frequency hopping signal in compressed domain [J]. Journal of Terahertz Science and electronic information, 2015,01:122-129..

[7] Candès E J, The restricted isometry property and its implications for compressed sensing[J], Comptes Rendus Mathematique , 2008, 346(9): 589-592.

[8] Chen S, Donoho D, editors. Basis pursuit[C]. Signals, Systems and Computers, 19941994 Conference Record of the Twenty-Eighth Asilomar Conference on; 1994: IEEE.

[9] Tropp J A, Gilbert A C. Signal recovery from random measurements via orthogonal matching pursuit[J]. Information Theory, IEEE Transactions on, 2007, 53(12): 4655-4666. 